\title{
Tendências da indústria de equipamentos odontológicos no Brasil entre 1990 e 2002: notas prévias
}

\author{
Trends of the dentistry equipment industry \\ in Brazil between 1990 and 2002: previous notes
}

Marco Antonio Manfredini ${ }^{1}$

Carlos Botazzo 2

\footnotetext{
${ }^{1}$ Secretaria Municipal de Saúde de São Paulo. Programa de PósGraduação em Ciências, Área de Concentração Saúde Coletiva, CCD/SES-SP. Rua Santo Antônio 590, 1o andar, 01314-000 São Paulo SP. manfra@uol.com.br

2 Instituto de Saúde de São Paulo. Programa de Pós-Graduação em Ciências, CCD/SES-SP.
}

\begin{abstract}
The searched subject inserts in the field of the Economy of the Health and retakes the debate on the concept of industrial medical complex, formulated for Hésio Cordeiro, in 1980, that it considered the study of the relations between the production and the circulation of medicines, the organization of the practical doctor, the forms of state intervention in the sector and practical the concrete ones of individual consumption. It enters the initial results of the research project, are distinguished the following characteristics of the dentistry equipment industry, that differentiates them of the remain of this producing park: an important production carried through in the proper country, the remarkable participation of the exports in relation to the imports and the great amount of countries to where the products are exported. A point that deserves to be pointed is the divergence between the collected data together the governmental organisms and to the representative association of the industry.

Key words Industrial complex of the health, Industrial-medical complex, Economy of the health, Dentistry equipment
\end{abstract}

Resumo Este artigo visa analisar a tendência da indústria de equipamentos odontológicos no Brasil, no período entre 1990 e 2002. Inserido no campo da Economia da Saúde, o estudo se fundamenta no conceito de complexo médico-industrial, formulado originalmente por Hésio Cordeiro. O estudo é exploratório das tendências desta indústria, em face da pequena produção científica sobre o tema na área acadêmica. Através da revisão da bibliografia e da coleta de informações nos órgãos governamentais e da indústria, observam-se grandes disparidades nos dados existentes, diante das mudanças de classificações e divergências nas informações provenientes das diversas fontes. O estudo sugere que, mesmo com as limitações metodológicas detectadas, a indústria de equipamentos odontológicos no Brasil apresenta características que as diferenciam em vários aspectos do restante deste parque produtor de bens. Entre essas, destacam-se a participação expressiva das exportações em relação às importações; $a$ grande quantidade de países para onde são exportados os produtos; uma suficiente produção realizada no próprio país e a pequena participação do setor público nos canais de comercialização destes produtos.

Palavras-chave Complexo industrial da saúde, Complexo médico-industrial, Economia da saúde, Equipamentos odontológicos 


\section{Introdução}

O tema pesquisado no presente trabalho se insere no campo da Economia da Saúde. Sua proposta é analisar as tendências da indústria de equipamentos odontológicos no Brasil entre 1990 e 2002. A proposta do trabalho é recuperar a evolução deste conjunto de práticas econômicas e seu subseqüente impacto nos custos do financiamento à saúde, seja de origem pública ou privada. Há um discurso difuso entre gestores públicos e cirurgiões-dentistas, que responsabiliza a pequena inserção da Odontologia no campo dos serviços estatais, argumentando que a instalação de equipamentos para esta prática seria muito onerosa e que esta indústria seria extremamente dependente de importações.

O corte histórico estabelecido compreende o período subseqüente à promulgação da legislação infraconstitucional da Saúde, em especial as Leis Federais no 8.080/90 e o 8.142/90, e o término da gestão de Fernando Henrique Cardoso, em 31 de dezembro de 2002.

A importância do estudo para o campo da Saúde Coletiva decorre do fato de que na análise da organização de serviços de saúde, sejam públicos ou privados, o fator do financiamento é tema recorrente na atualidade. Diversos autores apontam para o crescente impacto dos gastos em saúde nas economias dos países e suas relações com o mercado. No presente estudo, observam-se gastos realizados pelos agentes públicos e privados no consumo de equipamentos odontológicos.

\section{Revisão da literatura}

O referencial teórico que orienta o presente estudo é o do conceito de complexo médico-industrial. Formulado por Cordeiro ${ }^{1}$, em estudo sobre o padrão de consumo de medicamentos no país, este conceito tem servido como orientador de diversos autores.

Ao analisar a indústria da saúde e o processo de articulação do complexo médico-industrial, Cordeiro destaca que [...] as indicações no plano abstrato-formal nos remetem, como alternativa metodológica, ao estudo das relações entre a produção e a circulação de medicamentos, a organização da prática médica, as formas de intervenção estatal no setor e as práticas concretas de consumo individual. Neste trabalho, provisoriamente, deixa-se de lado a formação dos recursos humanos, as articulações da escola médica com a indústria farmacêutica e as relações com o setor de equipamentos médicos ${ }^{1}$.

A periodização definida por Cordeiro foi entre 1966 e 1978, tendo como critério a constituição do Instituto Nacional de Previdência Social, pois [...] demarca a intervenção estatal no sentido de uma mutação na prática médica: $o$ aceleramento das transformações que conduzem à internalização das relações capitalistas de produção na prática médica ${ }^{1}$.

A formulação de Cordeiro aponta para a reflexão sobre como o complexo médico-industrial é determinante nas práticas de consumo de bens e serviços de saúde. Ao estudar o complexo industrial da saúde e a necessidade de um enfoque dinâmico na economia da saúde, Gadelha destaca que a perspectiva de Hésio Cordeiro [...] foi retomada por diversos autores, podendo-se citar o livro organizado por Negri e Giovanni (2001), cujos trabalhos abordam tanto uma visão geral do processo de mercantilização da saúde, subseqüente ao período da capitalização [...] quanto o estudo dos setores produtivos de bens e serviços que fazem parte do complexo, além de outros artigos ou trabalhos que procuram tratar das indústrias da saúde e/ou do sistema de inovação em saúde, de acordo com uma perspectiva econômica (Gadelha ${ }^{2}$ ).

Gadelha realça que [...] a lógica empresarial capitalista penetra de forma arrebatadora todos os segmentos produtivos, envolvendo tanto as indústrias que já operavam tradicionalmente nessas bases - como a farmacêutica e de equipamentos médicos - quanto segmentos produtivos que possuíam formas de organização em que era possível verificar a convivência de lógicas empresariais com outras que dela se afastavam, como a produção de vacinas e outros produtos biológicos, fitoderivados e a prestação de serviços de saúde ${ }^{2}$.

Gadelha destaca que o setor saúde constitui um espaço importante de inovação e de acúmulo de capital, propiciando oportunidades de investimento, renda e emprego, ou seja, um locus essencial de desenvolvimento econômico, e uma área que requer uma forte presença do Estado e da sociedade para compensar as forças de geração de assimetrias e de desigualdades associadas à operação de estratégias empresariais e de mercado.

O mesmo autor afirma que [...] a delimitação do complexo industrial da saúde constitui, mais do que uma simples seleção de setores de atividades a partir de sua linha de produto (como medicamentos e equipamentos médicos) ou 
da propriedade do capital (segmento privado), um corte analitico que representa um olhar diferenciado frente à forma tradicional de abordar o setor saúde, representando uma percepção da área como um conjunto interligado de produção de bens e serviços em saúde que se movem no contexto da dinâmica capitalista 2 .

Outra reflexão importante é a realizada por Temporão, ao discutir o mercado privado de vacinas no Brasil. Ao analisar a questão dos insumos (medicamentos, imunobiológicos, hemoderivados e equipamentos médico-hospitalares), o autor destaca que há uma produção acadêmica razoável, mas concentrada ao campo da política de medicamentos e indústria farmacêutica. Em relação aos imunobiológicos, o autor afirma que [...] são escassos os estudos sobre esta área de atuação do Estado, sobre sua especificidade e singularidades. Estas, determinadas pelas características dos bens produzidos (vacinas) historicamente ligados a uma concepção tradicional de saúde pública não considerando questões do mercado e da competição (Temporão ${ }^{3}$ ).

O livro conduzido por Negri \& Giovanni4 é outra fonte importante para o presente estudo. Os autores organizam artigos de diferentes pesquisadores, formando um perfil da realidade do setor saúde no Brasil nos anos 90, a partir de um estudo coordenado pelo Instituto de Economia (IE) e pelo Núcleo de Políticas Públicas (NEPP) da Unicamp.

$\mathrm{Na}$ introdução do referido livro, Braga e Silva apontam que as décadas de 1970 e 1980 ficaram conhecidas como as do momento de capitalização da medicina. Os autores descrevem este período como um processo em que o Estado provia e pagava a ampliação da demanda aos serviços médicos e aos produtos industriais vinculados ao setor e simultaneamente financiava os investimentos e contratava os serviços da rede privada, apoiando os empreendimentos capitalistas no setor (Braga \& Silva ${ }^{5}$ ).

A crítica a este modelo, segundo os autores, influiu no avanço das políticas públicas e auxiliou a concretizar as idéias que estruturaram os capítulos dedicados à área social da Constituição Federal de 1988. Ao caracterizar o sistema de saúde na década de 1990, Braga e Silva destacam neste período a ocorrência da mercantilização da saúde, a descentralização da atenção pública em direção à municipalização, a internacionalização da oferta de produtos médicoindustriais, o esgarçamento dos mecanismos de financiamento público e o atraso na regulação do setor privado 5 .
É oportuno se destacar o conceito de mercantilização da saúde, descrito pelos autores como [...] processo pelo qual a atenção médica passa a ser uma mercadoria "como outra qualquer" submetida às regras de produção, financiamento e distribuição de tipo capitalista. É a fase que, no Brasil, se segue à da capitalização da medicina antes mencionada. Naquela, o Estado ficava no centro do processo, financiando, criando demanda pelos serviços, centralizando as decisões e estratégias. Na mercantilização, o setor privado vai se autonomizando no financiamento, cuja expressão máxima são os seguros-saúde privados, na produção de serviços que já conta com uma base própria de acumulação em expansão. Além disso, multiplicam-se os tipos de empresas de saúde e o consumidor de serviços se encontra no mercado e não mais diante do serviço público. O Estado perde relativo controle do processo e tenta regulá-lo, firmar contratos, supervisionar, em outras palavras, "correr atrás" dos abusos do poder econômico e tentar domesticálos a favor do povo e da nação 5 .

Ao analisar a evolução do setor de insumos e equipamentos médico-hospitalares, laboratoriais e odontológicos no Brasil durante a década de 1990, Furtado e Souza afirmam que esta indústria se formou nos anos 50 e atingiu o seu ápice nos anos 70. Os autores reiteram que a industrialização por substituição de importações foi muito dinâmica em termos de oferta. Esse dinamismo prossegue na década de 1980, com taxas inferiores.

A década de 1990, segundo Furtado e Sou$\mathrm{za},[. .$.$] marca um corte importante com relação$ à evolução anterior. O processo de substituição de importações se esgota e, portanto, a incorporação de novos itens à pauta de produtos fabricados no país [...] (Furtado \& Souza6).

Os autores concluem que a década de 1990 foi marcada por transformações estruturais no funcionamento dos principais mercados de equipamentos. Citam a maior abertura do mercado interno aos produtos importados, com as importações crescendo muito acima das exportações. Estes dados reforçam a necessidade de se realizar o presente estudo, uma vez que a tendência da indústria de equipamentos odontológicos durante os anos 90 poderia se diferenciar em alguns aspectos do conjunto do setor.

No caso da prática odontológica no Brasil, o modelo hegemônico no século 20 se pautou pelo acesso predominante a serviços privados, pela escassa cobertura populacional prestada pela odontologia privada na forma de convê- 
nios, seguros-saúde, cooperativas e autogestões e pela precária pressão social sobre o papel do Estado nesse setor. Sob esta lógica, as políticas públicas assumem uma posição de inferioridade em relação às práticas de mercado. Desta forma, não ter direito à saúde bucal seria uma fatalidade a que estaríamos condenados inapelavelmente.

O discurso realizado no âmbito da corporação odontológica reforça tal concepção. O problema dos preços praticados nos serviços privados decorreria de "os produtos serem importados" e "os equipamentos serem muito caros".

A visão de que a odontologia seria um problema a ser resolvido no mercado foi raras vezes afrontada no último século. O entendimento de que saúde bucal é direito de cidadania e dever do Estado fica restrito a momentos episódicos como as Conferências de Saúde Bucal e os encontros municipais, estaduais e nacional de serviços públicos odontológicos.

Este posicionamento político é claramente minoritário no campo das entidades odontológicas, das universidades e mesmo dos serviços públicos. Desta forma, consumir "odontologia" seria um problema a ser equacionado pelo mercado.

\section{Metodologia}

O estudo situa-se no campo da Economia da Saúde e é exploratório das tendências de comportamento de mercado da indústria de equipamentos odontológicos, área com poucos estudos no País.

A pesquisa descritiva foi realizada através da revisão da bibliografia existente, da coleta de dados nos órgãos governamentais e da indústria envolvida com o objeto do estudo e de observações realizadas em feiras comerciais de congressos odontológicos. A revisão bibliográfica abrange as publicações realizadas no Brasil sobre o complexo médico-industrial e a indústria de equipamentos médico-odontológicos, no período estudado, sendo realizada por meio de consultas em livros, periódicos científicos, meios de imprensa, textos, anais de congressos, dissertações, teses e sítios na internet. A coleta de dados foi realizada nos orgãos governamentais e nos setores industriais, em especial na Associação Brasileira da Indústria de Artigos e Equipamentos Médicos, Odontológicos, Hospitalares e de Laboratórios (Abimo).
As mudanças nas formas de classificação foram problemas detectados no estudo. O Instituto Brasileiro de Geografia e Estatística (IBGE) adotou uma nova forma de classificação para o setor de equipamentos médicos. Até a década de 1990, o IBGE adotava uma classificação bastante detalhada do setor de equipamentos médicos, com quatro grupos de produtos.

A partir de meados dos anos 90, o instituto adotou uma nova forma de classificação, o Cadastro Nacional de Atividades Econômicas (CNAE), assimilando três destes grupos em apenas um - o de fabricação de aparelhos e instrumentos para usos médico-hospitalares, odontológico e de laboratórios e aparelhos ortopédicos. O quarto grupo, relativo a itens de material de consumo, foi transferido para o setor farmacêutico 6 . Esta mudança de classificação traz dificuldades para a obtenção e síntese de informações para o presente estudo.

Em relação ao comércio internacional, houve uma mudança na forma de classificação dos códigos de mercadorias, para efeito das relações comerciais. Entre janeiro de 1989 e dezembro de 1985, o Brasil adotava a Nomenclatura Brasileira de Mercadorias (NBM). A nomenclatura tinha por base o Sistema Harmonizado de Designação e Classificação de Mercadorias ( $\mathrm{SH}$ ), que é formado pelos seis primeiros dígitos da nomenclatura. O Brasil criava mais quatro dígitos além do $\mathrm{SH}$, fazendo com que a NBM tivesse dez dígitos.

A partir de janeiro de 1996, o Brasil adotou a Nomenclatura Comum do Mercosul (NCM), em conjunto com a Argentina, Paraguai e Uruguai. Para a composição da NCM, os países do Mercosul agregaram dois dígitos de identificação de mercadorias aos seis da $\mathrm{SH}$, totalizando oito dígitos. Só um terço dos códigos NCM e NBM tem correlação direta entre si, pois no ato da criação da NCM, diversos códigos foram suprimidos ou sofreram desdobramentos (Brasil7).

Furtado \& Souza 6 salientam que a NBM era mais detalhada e completa, embora existissem algumas omissões como a falta de diferenciação entre as várias modalidades de aparelhos de ultra-som. Ao agrupar segmentos de equipamentos com outros que não eram equipamentos médicos, a NCM excluiu importantes segmentos, segundo os autores 6 .

Outra adversidade detectada no estudo foi a discrepância entre as informações veiculadas pelo setor industrial, pelo governo e pela mídia. É digna de registro esta constatação, uma vez que explicita a dificuldade na obtenção de 
informações que mensurem confiavelmente este mercado no País.

\section{Resultados}

Segundo Gutierrez \& Alexandre, o início da indústria nacional de insumos e equipamentos de uso médico estaria datado à década de 1950, quando são instaladas empresas de materiais de consumo, produtoras de artigos de pouca complexidade, como seringas e agulhas, e fabricantes de aparelhos de anestesia (Gutierrez \& Andrade 8 ). É oportuno se ressaltar que, no caso dos equipamentos odontológicos, a Indústria Brasileira de Aparelhos Dentários foi fundada em Ribeirão Preto em 1946, portanto em um momento anterior à caracterização do início da industrialização médica no País.

Ao definir o segmento odontológico na caracterização do setor de insumos e equipamentos médicos, Gutierrez e Alexandre 8 apontam como componentes os equipamentos, instrumental e materiais utilizados na prática odontológica. Na classificação de equipamentos, os autores destacam as cadeiras de dentista, equipos, refletores, equipamentos de raios $\mathrm{X}$, mochos, dosador e misturador de amálgamas, etc. 8

Devido à dificuldade na obtenção de dados no período estudado, observam-se lacunas importantes nas informações coletadas, o que gerou datas e períodos diversos, alguns sem a necessária informação.

Segundo a Abimo, no contexto de todas as empresas associadas a esta entidade, as indústrias odontológicas apresentaram, entre 1991 e 1996, uma participação percentual, conforme descrito na tabela 1.

Pode-se constatar que no período inicial do estudo, o total de empresas produtoras de equipamentos odontológicos no interior da Abimo oscilou de $11,5 \%$ a $19,8 \%$.

Em relação aos canais de comercialização no setor, a Abimo subdivide a informação, a partir de 2000, sob a forma de comercialização para o setor privado, setor público, exportações e outros (cessão de equipamentos por conta de contratos exclusivos de fornecimento de insumos e serviços, leasing, etc.).

Ao se compararem os canais de comercialização do setor odontológico de 2000 a 2002 , observa-se a situação apresentada na tabela 2 .

A análise destes dados aponta para uma participação expressiva do setor privado nas aquisições de equipamentos odontológicos. Dentre os seis segmentos estudados pela Abimo (odontologia, laboratório, radiologia, equipamento médico-hospitalar, implantes e materiais de consumo), a participação da comercialização privada na odontologia ocupava a segunda colocação, só sendo superada pela radiologia.

Por outro lado, o setor que teve a menor comercialização para o setor público em termos percentuais foi o odontológico. Para efeito de comparação, em 2001, do total de equipamentos comercializados pela Abimo no setor saúde, o setor público respondeu por $44,3 \%$ das compras (Abimo ${ }^{9}$ ), enquanto na área de equipamentos odontológicos, esta participação foi de apenas $13,4 \%$.

É oportuno se resgatar que a predominância do gasto privado no mercado de equipamentos odontológicos se mantém, mesmo com as várias inovações propostas para a organização dos ambientes de trabalho nos serviços públicos e a expansão da rede de serviços odontológicos no âmbito do Sistema Único de Saúde (SUS).

A Pesquisa de Assistência Médico-Sanitária do IBGE de 2002 apontava a existência de 24.129 equipos odontológicos e de 2.553 aparelhos de raios $\mathrm{X}$ odontológicos disponibilizados ao SUS. A distribuição por regiões brasileiras na ocasião está descrita no quadro 1 .

Na tabela 3 observa-se a distribuição percentual entre as regiões brasileiras, cotejandose com a distribuição da população.

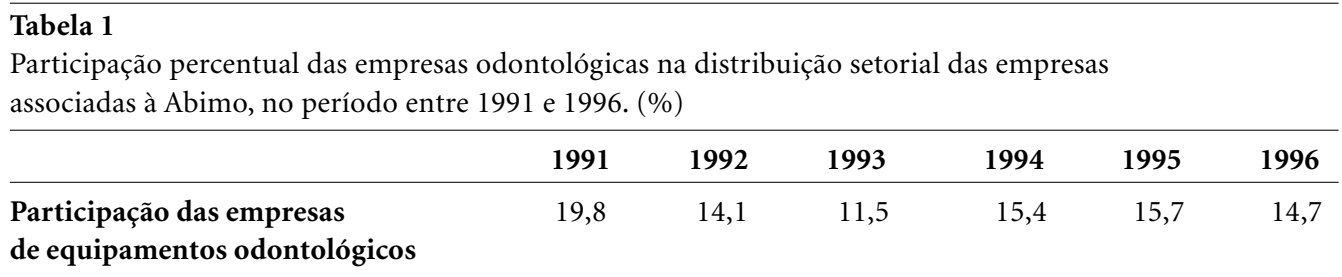

Fonte: Tabela elaborada por Manfredini, a partir de Abimo e Panorama Setorial12. 
Tabela 2

Canais de comercialização de equipamentos odontológicos, em porcentagem segundo a Abimo, entre 2000 e 2002. (\%)

\begin{tabular}{lrrr}
\hline & $\mathbf{2 0 0 0}$ & $\mathbf{2 0 0 1}$ & $\mathbf{2 0 0 2}$ \\
\hline Privado & 70,0 & 72,8 & 75,8 \\
Público & 20,0 & 13,4 & 12,2 \\
Exportações & 9,8 & 13,8 & 13,4 \\
Outros & 0,2 & & 1,5 \\
\hline
\end{tabular}

Fonte: Tabela elaborada por Manfredini,

a partir de Abimol1, 13 .

\section{Quadro 1}

Distribuição de equipos odontológicos e de aparelhos de raio $\mathrm{X}$ odontológicos, disponíveis ao SUS, segundo as regiões brasileiras, em 2001.

\begin{tabular}{lcc}
\hline Regiões & $\begin{array}{c}\text { Equipos } \\
\text { odontológicos }\end{array}$ & $\begin{array}{c}\text { Aparelhos de raio X } \\
\text { odontológicos }\end{array}$ \\
\hline Sudeste & 10.026 & 1.511 \\
Nordeste & 6.013 & 391 \\
Sul & 5.015 & 332 \\
Centro-Oeste & 1.878 & 145 \\
Norte & 1.197 & 154 \\
Total & $\mathbf{2 4 . 1 2 9}$ & $\mathbf{2 . 5 3 3}$ \\
\hline
\end{tabular}

Fonte: Quadro elaborado por Manfredini, a partir de IBGE14. gressos organizados pelas entidades odontológicas passam a ser espaços privilegiados para a divulgação de novos equipamentos, materiais de consumo e técnicas. Na maior parte destes eventos, a atualização técnico-científica ocupa um espaço físico mínimo ante as feiras comerciais acopladas.

Visitas a estes congressos podem se constituir em importantes momentos para a obtenção de informações e formulação de análises sobre o estado da arte do "complexo odontológico-industrial”. Nestes, nota-se uma crescente padronização na forma de apresentação dos consultórios odontológicos. Ao se visitarem os diversos stands, vê-se uma similaridade dos produtos ofertados pelos diversos fabricantes e que a dependência tecnológica de equipamentos importados se concentra na área de radiologia odontológica

Em relação ao comércio exterior, Furtado \& Souza 6 apresentam dados obtidos a partir de levantamento realizado na Secretaria de Comércio Exterior (Secex), entre 1990 e 1998. Segundo estes autores, as importações e exportações de equipamentos odontológicos comportaram-se, no período, conforme o descrito no quadro 2.

Segundo os dados da Secex, vê-se que a balança foi superavitária entre 1990 e 1995 e que, entre 1996 e 1998, as importações superam as exportações. Os autores destacam que no período de seu levantamento, os aparelhos odontológicos foram o único segmento em que houve equilíbrio entre as exportações e as importações, mesmo com o crescimento do valor das importações (Furtado \& Souza 6 ).

Ao analisar a evolução da balança comercial brasileira do segmento médico-hospitalar, entre 1999 e 2002, subdividindo-o em odontologia, laboratório, radiologia (diagnóstico por imagem), equipamentos médico-hospitalares, implantes e material de consumo, Gutierrez \& Alexandre 8 destacam que a odontologia foi o único segmento superavitário do setor. Os autores identificam como principais itens exportados as cadeiras odontológicas, aparelhos para dentistas e dentes artificiais. Os maiores itens importados foram materiais para restaurações, instrumentos e aparelhos e próteses dentárias ${ }^{8}$.

Por outro lado, a Abimo afirma que os valores relativos às exportações de equipamentos odontológicos entre 1992 e 2002 foram os que estão detalhados no quadro 3.

Vê-se que há uma grande divergência entre os valores obtidos por Furtado e Souza na Se- 
Tabela 3

Distribuição percentual da população, equipos odontológicos e de aparelhos de raio X odontológicos, disponíveis ao SUS, segundo as regiões brasileiras, em 2001. (\%)

\begin{tabular}{lrcc}
\hline Regiões & População & Equipos odontológicos & $\begin{array}{c}\text { Aparelhos de raio X } \\
\text { odontológicos }\end{array}$ \\
\hline Sudeste & 42,64 & 41,56 & 59,66 \\
Nordeste & 28,04 & 24,92 & 15,43 \\
Sul & 14,76 & 20,78 & 13,11 \\
Centro-Oeste & 6,89 & 7,78 & 5,72 \\
Norte & 7,67 & 4,96 & 6,08 \\
Total & $\mathbf{1 0 0 , 0 0}$ & $\mathbf{1 0 0 , 0 0}$ & $\mathbf{1 0 0 , 0 0}$ \\
\hline
\end{tabular}

Fonte: Tabela elaborada por Manfredini, a partir de IBGE14, 15.

\section{Quadro 2}

Importações e exportações de equipamentos odontológicos no Brasil entre 1990 e 1998, segundo a Secex, em dólares.

\begin{tabular}{lccr}
\hline Ano & Exportações (US\$) & Importações (US\$) & \multicolumn{1}{c}{ Saldo } \\
\hline 1990 & 8.458 .524 & 2.866 .066 & 5.592 .458 \\
1991 & 10.668 .991 & 2.289 .215 & 8.379 .776 \\
1992 & 12.608 .836 & 1.996 .775 & 10.612 .061 \\
1993 & 13.285 .034 & 2.944 .043 & 10.340 .991 \\
1994 & 15.537 .939 & 3.561 .411 & 11.976 .528 \\
1995 & 8.567 .368 & 7.640 .560 & 926.808 \\
1996 & 9.739 .016 & 12.266 .952 & $(-) 2.527 .936$ \\
1997 & 8.458 .524 & 13.423 .439 & $(-) 4.964 .915$ \\
1998 & 10.668 .991 & 12.300 .661 & $(-) 1.631 .670$ \\
\hline
\end{tabular}

Fonte: Quadro elaborado por Manfredini, a partir de Furtado \& Souza ${ }^{6}$.

cex e os disponibilizados pela Abimo. Sobressai-se a imprecisão das fontes, que empregam critérios distintos, o que impede a comparação entre os dados coletados.

Ao estudar a presença da concorrência dos importados e a participação de insumos provenientes do exterior nos custos de produção em empresas de saúde em Ribeirão Preto, Telles descreve que, no setor odontológico, a média de concorrência dos importados a este segmento seria de $14 \%$, variando de $5 \%$ para a fabricação de peças de mão e consultórios a $90 \%$ no mercado de microaparelhos odontológicos.

Em relação à participação dos insumos importados no custo de produção, Telles destaca, entre outros, a aquisição de silicone para manequins bucais, que representa $60 \%$ dos custos. A autora afirma que as empresas de equipamentos odontológicos utilizariam quase unicamente os insumos nacionais, com exceção da importação de rolamento para a fabricação das peças de mão, que podem resultar numa parti- cipação de $10 \%$ a $30 \%$ dos custos totais com insumos (Telles 10 ).

Em contatos mantidos com fabricantes e fornecedores em feiras comerciais de congressos odontológicos, observa-se que as áreas de maior dependência externa são as de maior incorporação tecnológica, em especial na área de imagens. Equipamentos radiológicos para exames extra-orais e imagens digitalizadas são na quase totalidade importados.

Ressalte-se entretanto que as informações disponibilizadas pelos dois maiores fabricantes de equipamentos odontológicos registrem que a exportação destes bens se processa para mais de 120 países no mundo (Gazeta Mercantil11).

\section{Conclusões}

A pesquisa aponta para a necessidade de construção de interfaces entre a Economia da Saúde e a Saúde Bucal. É notável que o campo da 
Quadro 3

Exportações de equipamentos odontológicos no Brasil, no período entre 1992 e 2002, em dólares.

\begin{tabular}{lc}
\hline Ano & Exportações (US\$) \\
\hline 1992 & 14.077 .360 \\
1993 & 11.441 .377 \\
1994 & 20.718 .718 \\
1995 & 21.998 .705 \\
1996 & 24.477 .792 \\
1997 & 24.961 .511 \\
1998 & 31.864 .039 \\
1999 & 25.417 .491 \\
2000 & 32.599 .131 \\
2001 & 29.045 .522 \\
2002 & 24.464 .538 \\
\hline
\end{tabular}

Fonte: Quadro elaborado por Manfredini, a partir de Abimo 16 .

Saúde Bucal Coletiva tenha construído diversas linhas de produção científica, mas tenha se esquivado do debate no campo da Economia da Saúde.

Pretende-se que, a partir das lacunas observadas no presente estudo, inicie-se uma linha de pesquisa que dê conta dos caracteres econômicos da prática odontológica. Mesmo com severas limitações, este estudo exploratório sugere que o País é um pujante produtor de equipamentos odontológicos, apresentando uma balança comercial superavitária no comércio exterior na maior parte do período estudado e elevada produção sustentada.
Portanto, a indústria de equipamentos odontológicos no Brasil apresenta características que as diferenciam em alguns aspectos do restante deste parque produtor de bens. Entre essas, destacam-se a participação expressiva das exportações em relação às importações; a grande quantidade de países para onde são exportados os produtos; uma suficiente produção realizada no próprio país e a pequena participação do setor público nos canais de comercialização destes produtos.

Como sugestão deste estudo, recomenda-se ao Ministério da Saúde que assuma o seu papel na regulação e no controle do parque industrial de equipamentos odontológicos no País. A omissão se estende ao controle da incorporação de novas tecnologias na área de saúde bucal e a ausência de uma política de avaliação sobre as tecnologias disponíveis e a criação de alternativas que possibilitem o desenvolvimento de tecnologias compatíveis com a realidade social brasileira.

$\mathrm{Na}$ lógica da Economia da Saúde, ressaltase a necessidade de elaboração de um cadastro único para o acompanhamento da produção de equipamentos no Brasil. Para tanto, sugerese que o Ministério da Saúde, o IBGE e a Abimo formulem uma proposição de classificação, que substitua o cadastro do IBGE de 1995. No que diz respeito ao comércio exterior, ressaltase a necessidade de se reavaliarem as nomenclaturas da Nomenclatura Comum do Mercosul, adotada a partir de janeiro de 1996.

\section{Colaboradores}

MA Manfredini e C Botazzo participaram igualmente de todas as etapas da elaboração do artigo. 


\section{Referências}

1. Cordeiro H. A indústria de saúde no Brasil. Rio de Janeiro: Graal, 1980.

2. Gadelha CAG. O complexo industrial da saúde e a necessidade de um enfoque dinâmico na economia da saúde. Rev C S Col 2003; 8(2):521-35.

3. Temporão JG. O mercado privado de vacinas no Brasil: a mercantilização no espaço da prevenção. Cad Saúde Pública 2003; 19(5):1323-39.

4. Negri B, Giovanni GD. Brasil: radiografia da saúde. Campinas: Unicamp, 2001.

5. Braga JCS, Silva PLB. Introdução: A mercantilização admissível e as políticas públicas inadiáveis: estrutura e dinâmica do setor saúde no Brasil. In: Negri B, Giovanni GD. Brasil: radiografia da saúde. Campinas: Unicamp; 2001. p. 19-42.

6. Furtado AT, Souza JH. Evolução do setor de insumos e equipamentos médico-hospitalares, laboratoriais e odontológicos no Brasil: a década de 90. In: Negri B, Giovanni GD. Brasil: radiografia da saúde. Campinas: Unicamp; 2001. p. 63-90.

7. Brasil. Ministério do Desenvolvimento, Indústria e Comércio Exterior. Correlação das Nomenclaturas. Brasília, 2005 [acessado 31/07/2005]. Disponível em www.desenvolvimento.gov.br/sitio/secex.

8. Gutierrez RMV, Alexandre PVM. Complexo industrial da saúde: uma introdução ao setor de insumos e equipamentos médicos. BNDES Setorial 2004; 19 (mar):119-55.

9. Associação Brasileira da Indústria de Artigos e Equipamentos Médicos, Odontológicos, Hospitalares e de Laboratórios. Perfil das indústrias. São Paulo: Abimo. Mimeo, 2003.
10. Telles LO. Clusters e a indústria ligada à área da saúde em Ribeirão Preto [dissertação]. São Paulo: Faculdade de Economia, Administração e Contabilidade, Universidade de São Paulo; 2002.

11. Gnatus e Dabi exportam mais equipamentos odontológicos. Gazeta Mercantil, 15/8/2005, p. C-2.

12. Análise setorial : sistema hospitalar. Panorama Setorial. Gazeta Mercantil, v. 2, dez. 1998.

13. Associação Brasileira da Indústria de Artigos e Equipamentos Médicos, Odontológicos, Hospitalares e de Laboratórios. A indústria de equipamentos médicos e hospitalares no Brasil. 2o Estudo Setorial. Instituto de Estudos e Marketing Industrial. São Paulo: Abimo; 2001.

14. Brasil. Ministério do Planejamento, Orçamento e Gestão. Instituto Brasileiro de Geografia e Estatística. IBGE. Estatísticas da saúde: assistência médicosanitária 2002 [acessado 9/07/2005]. Disponível em www.ibge.gov.br.

15. Brasil. Ministério do Planejamento, Orçamento e Gestão. Instituto Brasileiro de Geografia Estatística (IBGE). Estimativas populacionais com data de referência em 01 de julho dos respectivos anos e taxas médias geométricas de crescimento anual (\%) [acessado 13/06/2005]. Disponível em www.ibge.gov. br/download/estimativas.

16. Associação Brasileira da Indústria de Artigos e Equipamentos Médicos, Odontológicos, Hospitalares e de Laboratórios. Dados de exportações do odontológico, de 1992 a 2002. São Paulo: Abimo 2005 (informação prestada por Hely Audrey Maestrello, por e-mail em 30 de março de 2005).

Artigo apresentado em 5/07/2005

Aprovado em 30/08/2005

Versão final apresentada em 10/09/2005 